Fixed Point Theory, 21(2020), No. 2, 715-726

DOI: $10.24193 /$ fpt-ro.2020.2.51

http://www.math.ubbcluj.ro/ ${ }^{\text {nodeacj/sfptcj.html }}$

\title{
FOUR TO ONE
}

\author{
LECH PASICKI
}

AGH University of Science and Technology

Faculty of Applied Mathematics

Al. Mickiewicza 30

30-059 Kraków, Poland

E-mail: pasicki@agh.edu.pl

Abstract. In the present paper general theorems on a common fixed point for four mappings in dislocated metric space are proved. By the way, also results for three, two or one mapping are obtained. The assumptions are unified and compact. Numerous basic and sophisticated theorems can be derived from the facts presented in our paper.

Key Words and Phrases: Dislocated metric, weakly compatible mappings, fixed point.

2010 Mathematics Subject Classification: 54H25, 47H10.

Acknowledgements. This work was partially supported by the Faculty of Applied Mathematics AGH UST statutory tasks within subsidy of the Polish Ministry of Science and Higher Education, grant no. 11.11.420.004.

\section{REFERENCES}

[1] L.B. Ćirić, Generalized contractions and fixed-point theorems, Publ. Inst. Math. (Beograd), 12(1971), no. 26, 19-26.

[2] R.H. Haghi, Sh. Rezapour, N. Shahzad, Be careful on partial metric fixed point results, Topology Appl., 160(2013), 450-454.

[3] P. Hitzler, A. Seda, Mathematical Aspects of Logic Programming Semantics, Studies in Informatic Series, Chapman \& Hall, CRC Press, 2011.

[4] J. Jachymski, Equivalent conditions and the Meir-Keeler type theorems, J. Math. Anal. Appl., 194(1995), 293-303.

[5] G. Jungck, B.E. Rhoades, Fixed points for set valued functions without continuity, Indian J. Pure Appl. Math., 29(1998), 227-238.

[6] Z. Liu, X. Zhang, J.S. Ume, S.M. Kang, Common fixed point theorems for four mappings satisfying $\psi$-weakly contractive conditions, Fixed Point Theory Appl., 2015:20(2015).

[7] S.G. Matthews, Partial metric topology, Proc. 8th Summer Conference on General Topology and Applications, Ann. New York Acad. Sci., 728(1994), 183-197.

[8] D. O'Regan, N. Shahzad, R.P. Agarwal, Fixed point theory for generalized contractive maps on spaces with vector-valued metrics, Fixed Point Theory and Applications, (Eds. Y.J. Cho, J.K. Kim, S. M. Kang), Vol. 6, Nova Sci. Publ., New York, 2007, 143-149.

[9] L. Pasicki, Dislocated metric and fixed point theorems, Fixed Point Theory Appl., 2015:82(2015). 
[10] L. Pasicki, Four mappings and generalized contractions, Fixed Point Theory Appl., 2016:99(2016).

[11] L. Pasicki, Some extensions of the Meir-Keeler theorem, Fixed Point Theory Appl., 2017:1(2017).

[12] P.D. Proinov, Fixed point theorems in metric spaces, Nonlinear Anal., 64(2006), 546-557.

[13] I.A. Rus, A. Petruşel, G. Petruşel, Fixed Point Theory, Cluj University Press, 2008.

[14] I.A. Rus, A. Petruşel, M.A. Şerban, Weakly Picard operators: equivalent definitions, applications and open problems, Fixed Point Theory, 7(2006), no. 1, 3-22.

Received: June 21, 2018; Accepted: October 20, 2018. 